\title{
Autoimmune mechanisms in myasthenia gravis
}

\author{
Paola Cavalcante, Pia Bernasconi, and Renato Mantegazza
}

\begin{abstract}
Purpose of review
This article reviews recent findings on factors and mechanisms implicated in the pathogenesis of myasthenia gravis and briefly summarizes data on therapies acting at various stages of the autoimmune process.
\end{abstract}

\begin{abstract}
Recent findings
Data published over the last year promise to improve understanding of pathogenic mechanisms underlying myasthenia gravis. Animal studies have at last shown that antimuscle-specific kinase (MUSK) autoantibodies, like antiacetylcholine receptor (AChR) autoantibodies, are myasthenogenic. A new autoantigen, the low-density lipoprotein receptor-related protein 4 (LRP4), has been identified in variable proportions of otherwise seronegative patients. Anti-LRP4 antibodies may define a new myasthenia gravis subtype, supporting the concept that myasthenia gravis is not a single disease entity, and that different subtypes can differ in aetiology. Genetic and environmental factors are implicated in myasthenia gravis. The finding of persisting viral infection in the thymus of AChR-myasthenia gravis patients, combined with data on chronic inflammation, suggest that pathogens may favour intrathymic AChR-specific autosensitization and maintenance of autoimmunity in genetically susceptible individuals. Defective immunoregulatory mechanisms, involving pathogenic Th 17 and regulatory $T$ cells, contribute to tolerance loss and perpetuation of the autoimmune response in myasthenia gravis patients.
\end{abstract}

\section{Summary}

The recent identification of mechanisms initiating and perpetuating autoimmunity in myasthenia gravis may stimulate the development of more effective therapies.

\section{Keywords}

autoantibodies, autoimmunity, myasthenia gravis, thymus

\section{INTRODUCTION}

The pathogenic mechanisms causing autoimmunity in myasthenia gravis, an antibody-mediated disorder affecting the neuromuscular junction (NMJ), are incompletely understood. Most current treatments for myasthenia gravis do not lead to stable remission, highlighting the need to better understand events initiating and perpetuating the condition, in order to develop more effective therapies.

This review focuses on the following aspects of myasthenia gravis pathogenesis: autoimmune responses and associated clinical phenotypes; autoimmune processes at the NMJ; genetic and environmental triggers; intrathymic and peripheral mechanisms of loss of tolerance; and current and emerging therapies.

\section{MYASTHENIA GRAVIS: A PROTOTYPIC BUT MULTIFACETED AUTOIMMUNE DISEASE}

Myasthenia gravis is a prototypic humouralmediated autoimmune disease. Autoantibodies to the acetylcholine receptor (AChR) are present in approximately $80 \%$ of patients [1] and cause the typical muscle weakness and fatigability. Defining criteria for autoimmune diseases [2] are fulfilled; in particular, active immunization of animals with AChR leads to experimental myasthenia gravis (EAMG); IgG and complement are present at the $\mathrm{NMJ}$ in which AChR is reduced; and disease symptoms occur in mice on injection of IgG from myasthenia gravis patients $[1,3]$. However, the clinical myasthenia gravis features vary widely and

Department of Neurology IV, Neuromuscular Diseases and Neuroimmunology, Fondazione Istituto Neurologico 'Carlo Besta', Milan, Italy

Correspondence to Dr Renato Mantegazza, Department of Neurology IV, Neuromuscular Diseases and Neuroimmunology, Fondazione Istituto Neurologico 'Carlo Besta', Via Celoria 11, 20133 Milan, Italy. Tel: +39 02 23942372/2282; fax: +39 02 70633874; e-mail: rmantegazza@ istituto-besta.it

Curr Opin Neurol 2012, 25:621-629

DOI:10.1097/WCO.0b013e328357a829 


\section{KEY POINTS}

- A mechanism by which anti-MuSK autoantibodies exert their pathogenic effect at the NM has been described. If confirmed, this will have important implications for MuSK-myasthenia gravis therapy.

- The discovery of anti-LRP4 antibodies in a proportion of seronegative myasthenia gravis patients could lead to the identification of a new myasthenia gravis subtype and stimulate the search for specific therapies effective against this subtype.

- The autoimmune regulator AIRE appears to be involved in intrathymic immunoregulation by influencing the balance between pathogenic Th17 T cells and suppressive T-regulatory cells.

- Persisting viral infection has been identified in the thymus of AChR-positive myasthenia gravis patients, suggesting virus-mediated mechanisms in AChRmyasthenia gravis pathogenesis.

mechanisms leading to the production of anti-AChR antibodies are still unclear.

\section{Variation in clinical and autoimmune characteristics}

The heterogeneity of myasthenia gravis is manifest as follows:

(1) Variable autoantibody status, with some patients positive to antibodies against muscle endplate proteins other than AChR: approximately 5\% have antibodies to muscle-specific kinase (MuSK) and approximately 10\% are negative for AChR and MuSK (seronegative) [1]; approximately $66 \%$ of seronegative cases may have antibodies to clustered AChRs [4].

(2) Variable age of onset, with a female-dominant peak at less than 40 years (early-onset, EOMG) and male-dominant peak at more than 40 years (late-onset, LOMG) [5].

(3) Wide spectrum of symptoms, from ocular to generalized forms, with variation in disease progression [1].

(4) Variable thymic involvement. Although 50$60 \%$ of AChR-positive EOMG patients and some seronegative patients have follicular hyperplasia, and $10-15 \%$ of usually LOMG patients have thymoma, thymic disorder is absent from most MuSK-myasthenia gravis, and $10-20 \%$ of LOMG $[6,7]$.

(5) Variable treatment response, mainly in relation to variable disease severity, but likely also to patient-specific genetic factors $\left[8,9^{*}\right]$.
The clinical variability of myasthenia gravis allows classification into subtypes usually defined by autoantibody status, age at onset and thymic histology (Table 1), and suggests variation in pathogenic mechanisms. Although presence of anti-AChR antibody is useful for diagnosis, disease severity does not correlate with anti-AChR titre [10]. MuSK-myasthenia gravis patients have severe symptoms, with bulbar and respiratory muscle involvement, and resistance to therapy [11], whereas AChR-positive and seronegative patients frequently have generalized weakness [2].

A recent study found that peripheral blood lymphocytes from seronegative myasthenia gravis patients had proliferative responses to $\mathrm{AChR} \alpha$ subunit peptides $\left[12^{*}\right]$, suggesting that T-cell proliferation assay can usefully monitor AChR-specific autoimmune response in seronegative patients who have anti-AChR antibodies not detected by routine assay [4]. Most patients with thymoma have antibodies against titin, the ryanodine receptor, and the Kv1.4 $\alpha$-subunit of voltage-gated potassium channels, as well as AChR. These autoantibodies are normally associated with severe disease $[13,14]$ but a recent series of Kv1.4-positive Caucasian patients, mostly women, had mild LOMG, indicating that anti-Kv1.4 antibodies alone are not markers of severe myasthenia gravis, although they may predict lethal cardiac dysfunction and myocarditis [15"].

Three recent independent studies [16"'-18"'] identified a new myasthenia gravis autoantigen, low-density lipoprotein receptor-related protein 4 (LRP4) - an agrin receptor that activates MuSK and participates in AChR clustering $[19,20]$. Variable proportions of seronegative myasthenia gravis populations from Greece, the US, Germany, and Japan have anti-LRP4 antibodies.

Antibodies to aquaporin 4 - the autoantigen in neuromyelitis optica expressed in the central nervous system (CNS) and at the NMJ - have been identified in myasthenia gravis patients with CNS involvement $\left[21^{\prime}, 22^{\prime \prime}\right]$.

\section{Autoimmune processes at the neuromuscular junction}

AChR autoantibodies bind various subunits of the receptor, although most are specific for the main immunogenic region of the AChR $\alpha$ subunit [23] that is targeted in acute and chronic EAMG [24"]. The foetal AChR $\gamma$ subunit, expressed in extraocular muscles, may also induce an initially ocular autoimmune response progressing to generalized weakness [25]. Anti-AChR antibodies impair neuromuscular transmission mainly via 
Table 1. Summary of autoimmune responses and clinical features in myasthenia gravis subtypes

\begin{tabular}{|c|c|c|c|c|}
\hline & AChR-MG without thymoma & AChR-MG with thymoma & MuSK-MG & Seronegative-MG \\
\hline $\begin{array}{l}\text { Age at disease } \\
\text { onset }\end{array}$ & $\begin{array}{l}\text { Usually }<40 \text { years (EOMG); } \\
\text { Less offen }>40 \text { years (LOMG) }\end{array}$ & Usually >40 years (LOMG) & $\begin{array}{l}\text { Usually }<40 \text { years } \\
\text { (EOMG) }\end{array}$ & $\begin{array}{l}\text { Usually }<40 \text { years } \\
\text { (EOMG) }\end{array}$ \\
\hline Sex & Predominantly women & Male $:$ female ratio $=\sim 1: 1$ & Predominantly women & Predominantly women \\
\hline Autoantigens & $\begin{array}{l}\text { AChR in EOMG; AChR, titin, } \\
\text { and RyR in LOMG }\end{array}$ & $\begin{array}{l}\text { AChR, titin, RyR, Kvl.4, } \\
\text { and other muscle proteins }\end{array}$ & MuSK & $\begin{array}{l}\text { Abs not identified or } \\
\text { low-affinity; } \\
\text { anti-AChR Abs } \\
\text { ( 66\%) or Abs to } \\
\text { LRP4 (variable \%) }\end{array}$ \\
\hline $\begin{array}{l}\text { Autoimmune } \\
\text { processes } \\
\text { at NMJ }\end{array}$ & $\begin{array}{l}\text { Complement activation, endocytosis } \\
\text { of cross-linked AChR molecules } \\
\text { or blockage of AChR binding } \\
\text { site by anti-AChR Abs }\end{array}$ & $\begin{array}{l}\text { Complement activation, } \\
\text { endocytosis of cross-linked } \\
\text { AChR molecules or blockage } \\
\text { of AChR binding site by } \\
\text { anti-AChR Abs }\end{array}$ & $\begin{array}{l}\text { Blocked binding of } \\
\text { collagen Q to MuSK, } \\
\text { no complement } \\
\text { involvement }\end{array}$ & $\begin{array}{l}\text { Unknown; Role of } \\
\text { anti-LRP4 Abs } \\
\text { unknown }\end{array}$ \\
\hline Clinical presentation & $\begin{array}{l}\text { Ocular form at onset that frequently } \\
\text { progresses to generalized }\end{array}$ & $\begin{array}{l}\text { Generalized form with or } \\
\text { without bulbar involvement }\end{array}$ & $\begin{array}{l}\text { Generalized MG with } \\
\text { bulbar involvement } \\
\text { and frequent crises }\end{array}$ & $\begin{array}{l}\text { Similar to AChR-MG } \\
\text { without thymoma }\end{array}$ \\
\hline $\begin{array}{l}\text { Conventional } \\
\text { treatments }\end{array}$ & $\begin{array}{l}\text { Anti-AChE, immunosuppression or } \\
\text { thymectomy }\end{array}$ & $\begin{array}{l}\text { Same as AChR-MG (thymectomy } \\
\text { necessary) }\end{array}$ & $\begin{array}{l}\text { Same as AChR-MG } \\
\text { (no thymectomy) }\end{array}$ & Same as AChR-MG \\
\hline Thymic histology & $\begin{array}{l}\text { Hyperplasia in most EOMG; } \\
\text { Thymic atrophy in most LOMG }\end{array}$ & $\begin{array}{l}\text { Thymoma (hyperplasia common } \\
\text { in nonneoplastic thymus) }\end{array}$ & $\begin{array}{l}\text { Thymus resembles } \\
\text { that of age-matched } \\
\text { controls }\end{array}$ & $\begin{array}{l}\text { Hyperplastic changes } \\
\text { in variable } \\
\text { proportions of cases }\end{array}$ \\
\hline
\end{tabular}

Abs, antibodies; AChR, acetylcholine receptor; EOMG, early-onset MG; Kv1.4, $\alpha$-subunit of voltage-gated potassium channel; LOMG, late-onset MG; LRP4, lowdensity lipoprotein receptor-related protein 4; MG, myasthenia gravis; MuSK, muscle specific tyrosine kinase; NM, neuromuscular junction; RyR, ryanodine receptor.

complement-mediated destruction of the postsynaptic membrane [3].

The pathogenesis of MuSK-myasthenia gravis has begun to emerge recently. Anti-MuSK antibodies were shown to be pathogenic in animal studies that documented both presynaptic and postsynaptic NMJ changes $\left[26^{*}, 27^{\bullet}\right]$. Noninvolvement of the complement pathway was demonstrated when immunization of C5-deficient mice with MuSK caused severe muscle weakness and NMJ defects [28"']. The likely binding site of anti-MuSK antibodies was identified by in-vitro assays and passive transfer of MuSK-IgG to mice [29"']: autoantibodies blocked collagen $\mathrm{Q}$ binding to MuSK compromising agrin-mediated AChR clustering. Anti-MuSK IgG4 are the main antibodies in MuSK-myasthenia gravis. Passive transfer of anti-MuSK IgG4 to mice disrupted postsynaptic membrane ultrastructure and electrophysiological function, indicating that anti-MuSK IgG4 is myasthenogenic on its own [30"].

A pathogenic role of anti-LRP4 autoantibodies is also likely, as they are mainly of the complement activator IgG1 subclass, inhibit interactions between agrin and LRP4, and reduce AChR clustering in in-vitro systems $\left[16^{-"}, 17^{-"}\right]$.

\section{Genetic and environmental triggers}

As with other autoimmune diseases, human leukocyte antigen (HLA) loci are implicated in myasthenia gravis susceptibility. The HLA-A1-B8-DR3 (8.1) haplotype has a strong but complex effect on phenotype in EOMG associated with thymic hyperplasia [31]. Some studies point to involvement of HLA-DQ alleles in myasthenia gravis: DQB1*0502 was associated with myasthenia gravis in an Italian population [32] and HLA-DR14-DQ5 may increase susceptibility to MuSK-myasthenia gravis [33]. A high resolution HLA-DR analysis of patients from Texas revealed HLA-DQ associations with EOMG not observed in Europe, suggesting that regional differences in genetic susceptibility may be affected by environmental factors interacting with HLA genes [34"].

Several non-HLA loci are implicated in myasthenia gravis, including interferon- $\gamma$ (IFN), cytotoxic lymphocyte-associated protein-4 (CTLA-4), the AChR $\alpha$ subunit (CHRNA1) [35], and protein tyrosine phosphatase nonreceptor-22 (PTPN22), a lymphocyte activation mediator. A large study on Italian myasthenia gravis patients showed that the PTPN22 rs2476601 allele, associated with several autoimmune conditions [36], and known to interfere with autoreactive B-cell removal, and increase T-cell and dendritic-cell responsiveness [37",38"], is not associated with either myasthenia gravis, presence of autoantibodies, thymus abnormality sex, or age of myasthenia gravis onset [39], contrasting with previous findings in a French population [40]. However, the PTPN22 variant rs2488457, that disrupts a promoter binding site for AP-4 
transcription factors, correlated with low antibody titres and mild disease, suggesting a role of this variant in myasthenia gravis [39].

Pathogens are major environmental candidates for driving/perpetuating autoimmunity. Immunological cross-reactivity between AChR peptides and viral/microbial proteins has been reported [41,42], but evidence for pathogenic molecular mimicry in vivo has never been obtained. Early studies on antiviral antibody differences between myasthenia gravis patients and controls produced contrasting results $[43,44]$. Early attempts to identify/isolate viruses from myasthenia gravis thymuses largely failed too $[45,46]$, although Epstein-Barr virus (EBV) DNA was found in a few myasthenia gravis hyperplastic and thymoma thymuses [47]. More recently, signs of HTLV-I and poliovirus infection were found in myasthenia gravis thymus $[48,49]$. Myasthenia gravis exacerbations are known to be triggered by infections, whereas influenza vaccines have recently been reported to be beneficial $\left[50^{\circ}\right]$. Cases in which myasthenia gravis developed after HIV infection have been reported [51]. Another recent study found that high serum levels of IgG against EBV nuclear antigen 1 were associated with EOMG [52"] indicating a role of EBV in myasthenia gravis, as in other autoimmune disorders.

Our finding of active EBV infection in hyperplastic myasthenia gravis thymuses led us to suggest EBV involvement in the development/maintenance of the intrathymic autoimmune response [53,54"]. Two recent studies failed to identify EBV in hyperplastic thymuses $\left[55^{\circ}, 56^{\circ}\right]$ but detection sensitivity is likely to be an issue: it would help to reach a consensus on the most sensitive and reliable procedures for analysing EBV in thymus [57"].

\section{Intrathymic autoimmune mechanisms}

A wealth of data implicates the thymus as the main site of autosensitization in AChR-positive myasthenia gravis [58]. About $80 \%$ of AChR-positive patients have thymic abnormalities (hyperplasia or thymoma); hyperplastic thymus contains all elements necessary for initiating and maintaining an AChR-specific autoimmune response [autoantigen, expressed on thymic epithelial cells (TECs) and myoid cells; autoreactive T cells, and autoantibodyproducing B cells]; and stable remission occurs in high proportions of thymectomized patients.

Thymic hyperplasia is often of the follicular type and characterized by the presence of germinal centres containing B-cell infiltrates. The B cells undergo somatic hypermutation and antigendriven selection [59] and are surrounded by AChRexpressing myoid cells, suggesting germinal centres involvement in myasthenia gravis pathophysiology [60]. Upregulation of B-cell activating factor (BAFF) and a proliferation-inducing ligand in myasthenia gravis thymus suggests a microenvironment favourable to B-cell maturation/survival [61].

A combination of largely unknown factors likely leads to AChR autosensitization in the thymus of genetically susceptible individuals. Self-tolerance is normally achieved in thymus by negative selection of autoreactive T cells. Medullary TECs (mTECs) express tissue-specific antigens that select and permit deletion of autoreactive T cells [62]. Self-antigen expression is mediated by the autoimmune regulator (AIRE); AIRE loss-of-function mutations cause multiple-organ autoimmune polyglandular syndrome type I (APS-I), whereas AIRE deficiency impairs autoreactive T-cell deletion, resulting in autoimmunity in animals [63]. However, AIRE's role in human autoimmune diseases, including myasthenia gravis, is still unclear. As AIRE modulates CHRNA1 expression in human mTECs ex vivo, it has been proposed that, in the presence of specific CHRNA1 variants, AIRE sets the threshold for self-tolerance versus autoimmunity [35]. Although spontaneous myasthenia gravis is not a feature of AIRE $^{-/-}$mice, nor it is part of the APS-I syndrome in humans, a role of AIRE in the susceptibility of mice to EAMG and a relationship between FoxP3-expressing regulatory $\mathrm{T}$ cells (Tregs) and AIRE expression is suggested by recent data showing that $\mathrm{AIRE}^{-1-}$ mice have low Tregs and high expression of pathogenic Th17 in thymus [64"-]. Defective FoxP3 expression and severely impaired Treg function may also contribute to tolerance loss [65].

Chronic inflammation may also be involved in intrathymic pathogenesis of AChR-myasthenia gravis, by promoting immune-cell activation and increasing AChR expression in mTECs: hyperplastic myasthenia gravis thymus is characterized by high expression of inflammatory cytokines, IFNs, IFN-related genes, MHC class II genes, and lymphocyte-attractant chemokines [54',66]. The observation that in-vitro expression of AChR transcripts in myoid cells and TECs is increased by proinflammatory cytokines suggests how inflammation may contribute to intrathymic AChR-specific autosensitization [66].

In thymoma, lack of AIRE expression, absence of myoid cells, and failure to generate Tregs may all promote defective self-tolerance [67]. Recently, the expression of FoxP3 and the B-cell chemoattractant CXCL13 were found to vary with thymoma histology and myasthenia gravis subtype (generalized vs. ocular), prompting the proposal that different immunological processes underlie the two myasthenia gravis subtypes [68]. 
MuSK-positive patients display minimal thymic alterations, suggesting no pathogenic role of thymus in this type of myasthenia gravis.

\section{Intrathymic viral infection and innate immune system activation}

Dysregulated Toll-like receptor (TLR) signalling may drive/favour autoimmunity by inducing antigen presenting cell activation and inflammatory cytokine production, thereby priming adaptive immune cells. A recent study [69"] in multiple sclerosis (MS) patients supported previous findings [70] of latent EBV infection in active brain lesions overexpressing inflammatory IFN $\alpha$, and found that noncoding EBV RNA induced TLR3-mediated innate responses in cell culture, suggesting that EBV-driven innate immunity contributes to neuroinflammation in MS. TLR stimuli provide signals for B-cell maturation and survival - which significantly impact B-cell tolerance [71]. TLR activation may also influence self-tolerance via an effect on Tregs: in-vitro and in-vivo activation of dendritic cells by TLR7 ligands reduced Treg generation and suppressive function $\left[72^{-}\right]$.

The inflammatory signature of hyperplastic myasthenia gravis thymus is compatible with the hypothesis that persisting infection and TLRmediated innate immune activation are responsible for the chronic inflammatory state of thymus, the AChR autosensitization, and the ongoing autoimmune response (Fig. 1). Our previous finding that TLR4 is overexpressed in myasthenia gravis thymus [73] and our more recent discovery of TLR4-positive poliovirus-infected macrophages in some myasthenia gravis thymuses [49] suggest that pathogens eliciting innate immune responses might cause immunological alterations in the thymus, rendering the organ prone to autosensitization. As EBV is potentially able to immortalize autoreactive B cells, the presence of active EBV infection in the intrathymic lymphoid component of myasthenia gravis [53,54"] may provide a means of maintaining the autoimmune process. EBV itself might contribute to innate immune activation $\left[69^{-}, 74\right]$, lymphoid neogenesis or inflammation in myasthenia gravis thymus, whereas loss of tolerance of autoreactive cells inside and outside the thymus could be favoured by defective immunosuppression function of Tregs (Fig. 1).

\section{Altered immunoregulatory mechanisms}

Various peripheral immunomodulatory mechanisms, including suppression of autoreactive clones by Tregs, normally eliminate autoreactive cells not picked up centrally. Tregs are essential for selftolerance and defects in Tregs can cause experimental autoimmunity [75]. Imbalance between pathogenic Th17 and protective Tregs characterizes peripheral blood or target tissues in several autoimmune conditions [76-78], suggesting that altered immunoregulation contributes to autoimmunity.

Th17 cells affect autoantibody production by influencing the Th1/Th2-cytokine balance in myasthenia gravis peripheral blood mononuclear cells, which are characterized by reduced Treg number and FoxP3 expression [79]. Administration to EAMG rats of antibodies to interleukin 6 (IL-6) (a cytokine known to switch immune responses from Tregs induction to Th17 cells) suppresses ongoing myasthenia gravis through downregulation of Th17-related genes, and inhibits B cells and autoantibody production [80"-]. Administration of naïve $\mathrm{CD}^{+} \mathrm{CD} 25^{+}$Tregs to AChR-immunized animals prevents EAMG but does not improve ongoing EAMG, suggesting Treg involvement in controlling early events leading to EAMG [81]. Two recent studies reported higher serum IL-17 and IL-32 $\alpha$ (proinflammatory cytokines) in myasthenia gravis patients than controls. IL-17 levels correlated with anti-AChR antibody titres, whereas IL-32 $\alpha$ tended to decline with clinical improvement in generalized myasthenia gravis patients, suggesting pathogenic roles of these two cytokines $[82,83]$.

\section{Therapies targeting autoimmune mechanisms}

Long-term immunosuppression with corticosteroids is the mainstay treatment for autoimmune disorders. Corticosteroids are effective in most myasthenia gravis patients but do not produce permanent remission and have notable side effects, highlighting the need for improved therapies that target specific pathogenetic mechanisms. Other myasthenia gravis treatments are general immunosuppressants, such as azathioprine and cyclosporine, that preferentially inhibit T-cell proliferation and IL-2 production; cholinesterase inhibitors (e.g., pyridostigmine) that improve neuromuscular transmission by prolonging ACh availability at the NMJ; short-term immunomodulatory therapies (plasma exchange or intravenous immunoglobulins) that reduce circulating autoantibodies in patients with disease exacerbation; and thymectomy, which is effective in selected patients with pathologic or atrophic thymus $[8,84]$.

Recently, B-cell depletion with rituximab has been successful in myasthenia gravis, LambertEaton myasthenic syndrome $[85,86]$, and MuSKmyasthenia gravis. In the latter, rituximab produced remission or minimal manifestation status [87"]. 


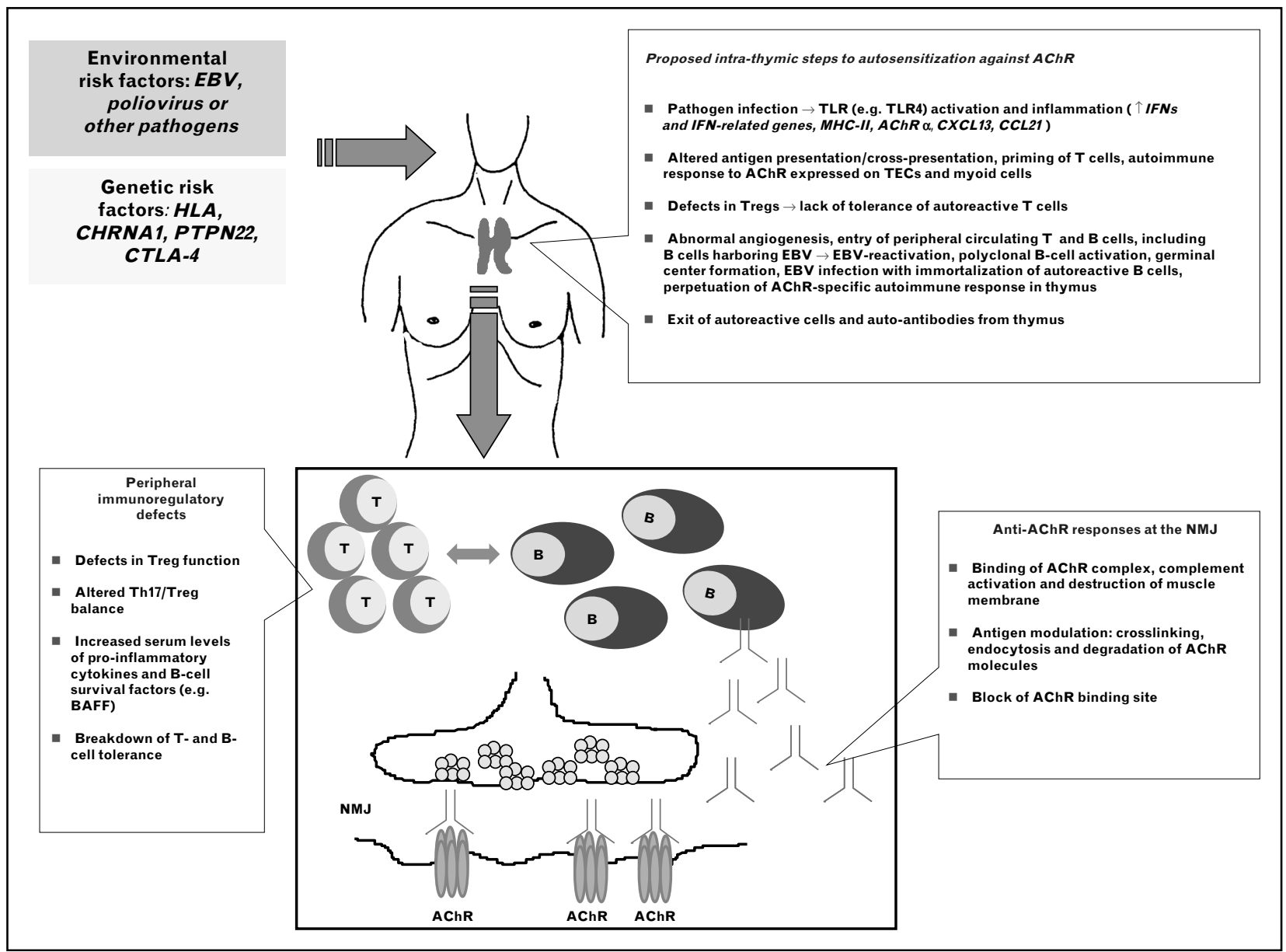

FIGURE 1. Proposed mechanisms for development and maintenance of autoimmune response in AChR-MG associated with nonneoplastic thymic abnormality. Dysregulated or persistent TLR activation by pathogens (particularly viral) or inflammatory triggers leads to the creation of a chronically inflamed thymic microenvironment, that, in the context of a predisposing genetic background, results in autosensitization to $\mathrm{AChR}$, in which professional antigen-presenting cells are activated and antigen cross-presentation by TECs/myoid cells is favoured, resulting in activation of AChR-specific T cells, which in turn promotes an autoimmune response by activated autoreactive B cells. Functionally deficient Tregs contribute to loss of tolerance of AChRspecific $T$ cells. By upregulating the production of T-cell and B-cell chemokines, the inflammatory state favours the recall of circulating $T$ and $B$ cells to thymus and their subsequent AChR-specific sensitization. B cells harbouring EBV are also attracted into the thymus, where EBV reactivation (in the inflamed microenvironment) results in propagation of EBV infection to uninfected cells and maintenance of the thymic autoimmune response, through immortalization of autoreactive B cells. AChRspecific $T$ and $B$ cells, and autoantibodies exit from the thymus to perpetuate the autoimmune response in the periphery, resulting in antibody attachment to AChR at the NM and impaired neuromuscular transmission. Altered peripheral immunoregulatory mechanisms, such as defective Th17/Treg balance, defective immunosuppressive function of Tregs and increased serum levels of proinflammatory cytokines and B-cell growth factors, may contribute to maintaining the AChR-specific autoimmune response. AChR, acetylcholine receptor; B, B cells/plasma cells; EBV, Epstein-Barr virus; IFN, interferon; MG, myasthenia gravis; NM, neuromuscular junction; T, T cells; TECs, thymic epithelial cells; TLR, Toll-like receptor.

However, this agent requires validation in randomized trials.

The proteasome inhibitor bortezomib, which depletes plasma cells and autoantibodies [88], and the complement pathway blocker anti-C1q antibody [89] have both been reported effective in EAMG. A recent pilot study using eculizumab, an anti-C5 complement monoclonal antibody, demonstrated a clinical benefit in refractory generalized myasthenia gravis patients [90], and belimumab, a monoclonal antibody inhibiting BAFF, has been proposed for a clinical trial which is not yet started.

Recent findings that EBV infection is a feature of myasthenia gravis thymus $\left[53,54^{-}\right]$suggest that antiviral agents and treatments targeting the innate 
immune system should be investigated. Vaccination (for myasthenia gravis prevention) and antiviral treatment for chronic EBV-related myasthenia gravis might also be worth trying.

\section{CONCLUSION}

Recent work has improved our understanding of pathogenic mechanisms in myasthenia gravis, but we are still not at a point where knowledge can drive the development of new treatments. Perhaps discovery of the probable pathogenic mechanism of anti-MuSK antibodies will change this. We believe it is important to focus on the environmental factors that, interacting with the genetic background, generate and maintain autoimmunity. One such factor appears to be active intrathymic EBV infection in AChR-positive myasthenia gravis patients. If confirmed, therapeutic approaches targeting EBV will need testing. This will involve assessing virological, serological, and immunological variables in relation to clinical course and response to therapy; and also performing epidemiologic studies to relate prior EBV exposure to subsequent myasthenia gravis and identify susceptible individuals.

\section{Acknowledgements}

P.C. is supported by 7th Framework Programme of the European Union FIGHT-MG (Grant No. 242210) and by Italian Ministry of Health, years 2011-2012 (annual research funding).

\section{Conflicts of interest}

P.B. has received a speaker honorarium from MEDA Pharmaceuticals Inc. R.M. has served on a scientific advisory board for GlaxoSmithKline, Alexion, and BioMarin and has received funding for travel and speaker honoraria from Sanofi-Aventis, Merck-Serono and MEDA Pharmaceuticals Inc.

\section{REFERENCES AND RECOMMENDED}

\section{READING}

Papers of particular interest, published within the annual period of review, have been highlighted as:

- of special interest

-1. of outstanding interest

Additional references related to this topic can also be found in the Current World Literature section in this issue (p. 647).

1. Vincent A. Unravelling the pathogenesis of myasthenia gravis. Nat Rev Immunol 2002; 2:797-804.

2. Rose NR, Bona C. Defining criteria for autoimmune diseases (Witebsky's postulates revisited). Immunol Today 1993; 14:426-430.

3. Conti-Fine BM, Milani M, Kaminski HJ. Myasthenia gravis: past, present, and future. J Clin Invest 2006; 116:2843-2854.

4. Leite Ml, Jacob S, Viegas S, et al. IgG1 antibodies to acetylcholine receptors in 'seronegative' myasthenia gravis. Brain 2008; 131:1940-1952.

5. Mantegazza R, Baggi $F$, Antozzi $C$, et al. Myasthenia gravis (MG): epidemiological data and prognostic factors. Ann NY Acad Sci 2003; 998:413-423.

6. Marx A, Wilisch A, Schultz A, et al. Pathogenesis of myasthenia gravis. Virchows Archiv 1997; 430:355-364.
7. Leite MI, Strobel $\mathrm{P}$, Jones $\mathrm{M}$, et al. Fewer thymic changes in MuSK antibodypositive than in MuSK antibody-negative MG. Ann Neurol 2005; 57:444448.

8. Mantegazza R, Bonanno S, Camera G, Antozzi C. Current and emerging therapies for the treatment of myasthenia gravis. Neuropsychiatr Dis Treat 2011; 7:151-160.

9. Colleoni L, Kapetis D, Maggi L, et al. A new thiopurine S-methyltransferase

- haplotype associated with intolerance to azathioprine. J Clin Pharmacol 2012 [Epub ahead of print].

In this study the authors identify a novel haplotype, designated TPMT $* 3 \mathrm{E}$, associated with intolerance to azathioprine.

10. Drachman DB, Adams RN, Josifek LF, Self SG. Functional activities of autoantibodies to acetylcholine receptors and the clinical severity of myasthenia gravis. N Engl J Med 1982; 307:769-775.

11. Guptill JT, Sanders DB, Evoli A. Anti-MuSK antibody myasthenia gravis: clinical findings and response to treatment in two large cohorts. Muscle Nerve $2011 ; 44: 36-40$.

12. Oshima $M$, Deitiker $P R$, Smith $R G$, et al. T-cell recognition of acetylcholine

- receptor provides a reliable means for monitoring autoimmunity to acetylcholine receptor in antibody-negative myasthenia gravis patients. Autoimmunity 2012; 45:153-160.

The study reports that $T$ cells from seronegative myasthenia gravis patients undergo proliferation in response to $\mathrm{AChR} \alpha$ subunit peptides, indicating that $\mathrm{T}$ cell proliferation assay can be used to monitor anti-AChR autoimmune response in seronegative patients.

13. Maggi L, Andreetta $F$, Antozzi $C$, et al. Thymoma-associated myasthenia gravis: outcome, clinical and pathological correlations in 197 on a 20 -year experience. J Neuroimmunol 2008; 202:237-244.

14. Suzuki S, Utsugisawa K, Nagane $Y$, Suzuki N. Three types of striational antibodies in myasthenia gravis. Autoimmune Dis 2011; 2011:740583.

15. Romi F, Suzuki S, Suzuki N, et al. Antivoltage-gated potassium channel Kv1.4 antibodies in myasthenia gravis. J Neurol 2012; 259:1312-1316.

This article provides evidence that anti-Kv1.4 antibodies are present in Caucasian patients with mild myasthenia gravis, indicating that these antibodies are not a marker of myasthenia gravis severity, at least in Caucasians.

16. Zhang B, Tzartos JS, Belimezi M, et al. Autoantibodies to lipoprotein-related

- protein 4 in patients with double-seronegative myasthenia gravis. Arch Neurol 2012; 69:445-451.

This was the first study to demonstrate the presence of anti-LRP4 antibodies in some seronegative myasthenia gravis patients from Greece and the US. This new myasthenia gravis autoantigen may identify a new myasthenia gravis subgroup with distinct clinical features perhaps requiring different treatment.

17. Higuchi $O$, Hamuro J, Motomura M, Yamanashi Y. Autoantibodies to low-

- density lipoprotein receptor-related protein 4 in myasthenia gravis. Ann Neurol $2011 ; 69: 418-422$.

This study demonstrates the presence of anti-LRP4 antibodies in Japanese seronegative myasthenia gravis patients. Like $\left[16^{\text {"n }}\right.$ ], it suggests a new myasthenia gravis subtype perhaps requiring different treatment.

18. Pevzner A, Schoser B, Peters $K$, et al. Anti-LRP4 autoantibodies in AChR- and muSK-antibody-negative myasthenia gravis. J Neurol 2012; 259:427-435. This study was the first to demonstrate anti-LRP4 antibodies in some seronegative myasthenia gravis patients from Germany. Like [16 $]$ and [17 $]$, it suggests a new myasthenia gravis subtype perhaps requiring different treatment.

19. Kim N, Stiegler AL, Cameron TO, et al. Lrp4 is a receptor for Agrin and forms a complex with MuSK. Cell 2008; 135:334-342.

20. Zhang B, Luo S, Wang $Q$, et al. LRP4 serves as a coreceptor of agrin. Neuron 2008; 60:285-297.

21. Vaknin-Dembinsky $A$, Abramsky $O$, Petrou $P$, et al. Myasthenia gravis-asso-

- ciated neuromyelitis optica-like disease: an immunological link between the central nervous system and muscle? Arch Neurol 2011; 68:1557-1561.

In some myasthenia gravis patients with overt CNS involvement, the autoimmune process also targeted aquaporin 4 (AQP4) (autoantigen in neuromyelitis optica), suggesting an immunological link between the CNS and muscles.

22. Jarius $S$, Paul F, Franciotta $D$, et al. Neuromyelitis optica spectrum disorders in

- patients with myasthenia gravis: ten new aquaporin-4 antibody positive cases and a review of the literature. Mult Scler 2012; 18:1135-1143.

New anti-AQP4 antibody-positive cases with myasthenia gravis and neuromyelitis optica (NMO) are presented, and the clinical features of patients with NMO spectrum disorders and myasthenia gravis are reviewed, emphasizing that myasthenia gravis patients with atypical motor or optic symptoms should be tested for AQP4 antibodies.

23. Vincent $A$, Willcox $N$, Hill $M$, et al. Determinant spreading and immune responses to acetylcholine receptors in myasthenia gravis. Immunol Rev 1998; 164:157-168.

24. Luo J, Lindstrom J. Myasthenogenicity of the main immunogenic region and

- endogenous muscle nicotinic acetylcholine receptors. Autoimmunity 2012; 45:245-252.

The main immunogenic region (MIR) of the $\mathrm{AChR} \alpha$ subunit was incorporated into Aplysia ACh-binding protein and investigated for its ability to induce EAMG. The initial autoimmune response, directed at MIR, apparently led to an autoimmune response sustained by muscle AChRs.

25. Wu X, Tuzun E, Li J, et al. Ocular and generalized myasthenia gravis induced by human acetylcholine receptor $\gamma$ subunit immunization. Muscle Nerve 2012; 45:209-216. 
26. Richman DP, Nishi K, Morell SW, et al. Acute severe animal model of - antimuscle-specific kinase myasthenia: combined postsynaptic and presynaptic changes. Arch Neurol 2012; 69:453-460.

Investigation of an acute severe model of MuSK-myasthenia gravis indicated that MuSK autoantibodies induce both presynaptic and postsynaptic changes at the NMJ.

27. Viegas $S$, Jacobson $L$, Waters $P$, et al. Passive and active immunization

- models of MuSK-Ab positive myasthenia: Electrophysiological evidence for pre and postsynaptic defects. Exp Neurol 2012; 234:506-512.

On the basis of the results of investigation of passive and active immunization models of MuSK-myasthenia gravis, MuSK antibodies are suggested first to compromise MuSK's ability to maintain a high density of AChRs at the postsynaptic membrane and second to interfere with a compensatory presynaptic mechanism that helps preserve NMJ function.

28. Mori $\mathrm{S}$, Kubo $\mathrm{S}$, Akiyoshi $\mathrm{T}$, et al. Antibodies against muscle-specific kinase

-1. impair both presynaptic and postsynaptic functions in a murine model of myasthenia gravis. Am J Pathol 2012; 180:798-810.

This study demonstrates that complement is not necessary for the development of MuSK-myasthenia gravis, an important milestone toward understanding how antiMuSK antibodies impair neuromuscular transmission.

29. Kawakami $Y$, Ito $M$, Hirayama $M$, et al. Anti-MuSK autoantibodies block - binding of collagen $\mathrm{Q}$ to MuSK. Neurology 2011; 77:1819-1826.

First indication of a specific binding site for anti-MuSK antibodies which block binding of collagen $\mathrm{Q}$ to MuSK, thereby compromising agrin-mediated AChR clustering. This finding is potentially of major importance for developing new specific treatments for MuSK-myasthenia gravis.

30. Klooster R, Plomp JJ, Huijbers MG, et al. Muscle-specific kinase myasthenia

- gravis IgG4 autoantibodies cause severe neuromuscular junction dysfunction in mice. Brain 2012; 135:1081-1101.

This study demonstrates that purified lgG4 from MuSK-myasthenia gravis patients binds mouse NMJ and causes severe muscle weakness, without requiring other immune system components. Electrophysiological investigations revealed severe reduction of postsynaptic acetylcholine sensitivity and depressed presynaptic acetylcholine release during intense activity, with no compensatory upregulation of transmitter release.

31. Vandiedonck C, Giraud M, Garchon HJ. Genetics of autoimmune myasthenia gravis: the multifaceted contribution of the HLA complex. J Autoimmun 2005; 25:6-11.

32. Baggi $F$, Antozzi $C$, Andreetta $F$, et al. Identification of a novel HLA class II association with DQB1*0502 in an Italian myasthenic population. Ann NY Acad Sci 1998; 841:355-359.

33. Niks EH, Kuks JB, Roep BO, et al. Strong association of MuSK antibodypositive myasthenia gravis and HLA-DR14-DQ5. Neurology 2006; 66:17721774.

34. Deitiker PR, Oshima M, Smith RG, et al. Association with HLA DQ of early

- onset myasthenia gravis in Southeast Texas region of the United States. Int J Immunogenet 2011; 38:55-62.

This study demonstrates new associations between HLA-DQ loci and early-onset myasthenia gravis in Southeast Texas patients, not previously reported in European myasthenia gravis patients, suggesting that region-specific environmental factors may interact with HLA genes to predispose toward myasthenia gravis.

35. Giraud M, Taubert R, Vandiedonck $C$, et al. An IRF8-binding promoter variant and AIRE control CHRNA1 promiscuous expression in thymus. Nature 2007; 448:934-937.

36. Gregersen PK, Lee HS, Batliwalla F, Begovich AB. PTPN22: setting thresholds for autoimmunity. Semin Immunol 2006; 18:214-223.

37. Menard L, Saadoun D, Isnardi I, et al. The PTPN22 allele encoding an R620W variant interferes with the removal of developing autoreactive $B$ cells in humans. J Clin Invest 2011; 121:3635-3644.

This article demonstrates that the R620W variant of PTPN22 interferes with the removal of autoreactive $B$ cells and provides an explanation for the R620W variant's known role in conferring increased risk of developing autoimmunity.

38. Zhang J, Zahir N, Jiang Q, et al. The autoimmune disease-associated PTPN22

- variant promotes calpain-mediated Lyp/Pep degradation associated with lymphocyte and dendritic cell hyperresponsiveness. Nat Genet 2011; 43:902-907.

This study demonstrates that the PTPN22 R620W variant is able to promote hyperresponsiveness in lymphocytes and dendritic cells, thus providing a functional explanation for the previously observed genetic association of the R620W variant with different autoimmune diseases.

39. Provenzano C, Ricciardi R, Scuderi F, et al. PTPN22 and myasthenia gravis: replication in an Italian population and meta-analysis of literature data. Neuromuscul Disord 2012; 22:131-138.

40. Vandiedonck C, Capdevielle $C$, Giraud $M$, et al. Association of the PTPN22*R620W polymorphism with autoimmune myasthenia gravis. Ann Neurol 2006; 59:404-407.

41. Schwimmbeck PL, Dyrberg T, Drachman DB, Oldstone MBA. Molecular mimicry and myasthenia gravis. An autoantigenic site of the acetylcholine receptor alpha-subunit that has biologic activity and reacts immunochemically with herpes simplex virus. J Clin Invest 1989; 84:1174-1180.

42. Marino Gammazza A, Bucchieri F, Grimaldi LM, et al. The molecular anatomy of human Hsp60 and its similarity with that of bacterial orthologs and acetylcholine receptor reveal a potential pathogenetic role of antichaperonin immunity in myasthenia gravis. Cell Mol Neurobiol 2012; 32:943-947.
43. Tindall RSA, Cloud R, Lucky J, Rosenberg RN. Serum antibodies to cytomegalovirus in myasthenia gravis: effects of thymectomy and steroids. Neurology 1978; 28:273-277.

44. Klavinskis LS, Willcox N, Newsom-Davis J. Antivirus antibodies in myasthenia gravis. Neurology 1985; 35:1381-1384.

45. Aoki $\mathrm{T}$, Drachman $\mathrm{DB}$, Asher DM, et al. Attempts to implicate viruses in myasthenia gravis. Neurology 1985; 35:185-192.

46. Klavinski LS, Willcox HNA, Richmond JE, Newsom-Davis J. Attempted isolation of viruses from myasthenia gravis thymus. J Neuroimmunol 1986; 11:287-299.

47. McGuire $L$, Huang DP, Teoh $R$, et al. Epstein-Barr virus genome in thymoma and thymic lymphoid hyperplasia. Am J Pathol 1988; 131:385-390.

48. Manca N, Perandin F, De Simone N, et al. Detection of HTLVI tax-rex and pol gene sequences of thymus gland in large group of patients with myasthenia gravis. J Acquir Immune Defic Syndr 2002; 29:300-306.

49. Cavalcante $P$, Barberis $M$, Cannone $M$, et al. Detection of poliovirus-infected macrophages in thymus of patients with myasthenia gravis. Neurology 2010; $74: 1118-1126$.

50. Auriel E, Regev K, Dori $A$, Karni $A$. Safety of influenza and $H 1 N 1$ vaccinations

- in patients with myasthenia gravis, and patient compliance. Muscle Nerve $2011 ; 43: 893-894$.

This study provides data suggesting that seasonal influenza and $\mathrm{H} 1 \mathrm{~N} 1$ vaccination is well tolerated in myasthenia gravis and can prevent infection-associated myasthenia gravis exacerbations.

51. Hung WL, Lin $Y H$, Wang $P Y$, Chang $M H$. HIV-associated myasthenia gravis and impacts of HAART: one case report and a brief review. Clin Neurol Neurosurg 2011; 113:672-674.

52. Csuka D, Banati $M$, Rozsa $C$, et al. High anti-EBNA-1 lgG levels are - associated with early-onset myasthenia gravis. Eur J Neurol 2012; 19: 842-846.

A supposed association between Epstein-Barr virus (EBV) infection and earlyonset myasthenia gravis, is supported by evidence that high levels of EBV nuclear antigen (EBNA)-1 antibodies are more common in myasthenia gravis than healthy controls and are particularly associated with early onset myasthenia gravis.

53. Cavalcante $P$, Serafini $B$, Rosicarelli $B$, et al. Epstein-Barr virus persistence and reactivation in myasthenia gravis thymus. Ann Neurol 2010; 67:726738.

54. Cavalcante $P$, Maggi $L$, Colleoni $L$, et al. Inflammation and Epstein-Barr virus

- infection are common features of myasthenia gravis thymus: possible roles in pathogenesis. Autoimmune Dis 2011; 2011:213092.

This study supports and extends previous data on inflammation and active EBV infection in hyperplastic myasthenia gravis thymus, and suggests that EBV infection and chronic inflammation may play major roles in intrathymic maintenance of the autoimmune response in myasthenia gravis.

55. Kakalacheva K, Maurer MA, Tackenberg B, et al. Intrathymic Epstein-Barr virus infection is not a prominent feature of myasthenia gravis. Ann Neurol 2011; 70:508-514.

This study reports absent or very low levels of EBV in thymuses of early-onset myasthenia gravis patients, leading the authors to conclude that EBV has no aetiologic role in early-onset myasthenia gravis.

56. Meyer M, Höls AK, Liersch $B$, et al. Lack of evidence for Epstein-Barr virus - infection in myasthenia gravis thymus. Ann Neurol 2011; 70:515-518.

Again absent or very low levels of EBV infection were reported in thymuses of earlyonset myasthenia gravis patients, leading the authors to conclude that there is no direct role of thymic EBV infection in myasthenia gravis pathogenesis.

57. Serafini $B$, Cavalcante $P$, Bernasconi $P$, et al. Epstein-Barr virus in myasthenia - gravis thymus: a matter of debate. Ann Neurol 2011; 70:519.

A debate on the methodological and investigational aspects in the analysis of EBV expression in myasthenia gravis patients is provided together with the comparison of BZLF1 expression, a protein associated with EBV lytic phase, between tonsil and thymus.

58. Cavalcante $P$, Le Panse $R$, Berrih-Aknin $S$, et al. The thymus in myasthenia gravis: site of 'innate autoimmunity'? Muscle Nerve 2011; 44:467-484.

59. Sims GP, Shiono H, Willcox N, Stott DI. Somatic hypermutation and selection of $B$ cells in thymic germinal centers responding to acetylcholine receptor in myasthenia gravis. J Immunol 2001; 167:1935-1944.

60. Roxanis I, Micklem K, McConville J, et al. Thymic myoid cells and germinal center formation in myasthenia gravis; possible roles in pathogenesis. J Neuroimmunol 2002; 125:185-197.

61. Thangarajh $M$, Masterman $T$, Helgeland $L$, et al. The thymus is a source of B-cell survival factors-APRIL and BAFF-in myasthenia gravis. J Neuroimmunol 2006; 178:161-166.

62. Kyewski $B$, Klein $L$. A central role for central tolerance. Annu Rev Immunol 2006; $24: 571-606$.

63. Akirav EM, Ruddle $\mathrm{NH}$, Herold $\mathrm{KC}$. The role of AIRE in human autoimmune disease. Nat Rev Endocrinol 2011; 7:25-33.

64. Aricha R, Feferman T, Scott HS, et al. The susceptibility of Aire(-/-) mice

-1 to experimental myasthenia gravis involves alterations in regulatory $T$ cells. J Autoimmun 2011; 36:16-24.

This study provides the first indication of a link between the autoimmune regulator AIRE and FoxP3-expressing regulatory T cells, and suggests involvement for both in myasthenia gravis pathogenesis. If confirmed this finding is likely to lead to better understanding of mechanisms leading to loss of tolerance of autoreactive T cells in myasthenia gravis. 
65. Balandina $A$, Lécart $S$, Dartevelle $P$, et al. Functional defect of regulatory CD4(+)CD25+ T cells in the thymus of patients with autoimmune myasthenia gravis. Blood 2005; 105:735-741.

66. Poëa-Guyon S, Christadoss $P$, Le Panse R, et al. Effects of cytokines on acetylcholine receptor expression: implications for myasthenia gravis. J Immunol 2005; 174:5941-5949.

67. Marx A, Willcox N, Leite Ml, et al. Thymoma and paraneoplastic myasthenia gravis. Autoimmunity 2010; 43:413-427

68. Zhang $\mathrm{M}$, $\mathrm{Li} \mathrm{H}$, Guo J, et al. Different molecular expression in thymoma with ocular or generalized myasthenia gravis. J Neurol Sci 2012; 313:27-31.

69. Tzartos JS, Khan G, Vossenkamper A, et al. Association of innate immune

- activation with latent Epstein-Barr virus in active MS lesions. Neurology 2012; 78:15-23.

This study supports previous findings that latent EBV is present in active MS lesions in brain and found Toll-like receptor-3-mediated innate immune response associated with EBV infection, suggesting that EBV-driven innate immune responses might contribute to neuroinflammation in the MS brain.

70. Serafini B, Rosicarelli B, Franciotta D, et al. Dysregulated Epstein-Barr virus infection in the multiple sclerosis brain. J Exp Med 2007; 204:2899-2912.

71. Crampton SP, Voynova E, Bolland S. Innate pathways to B-cell activation and tolerance. Ann N Y Acad Sci 2010; 1183:58-68.

72. Hackl D, Loschko J, Sparwasser T, et al. Activation of dendritic cells via TLR7

- reduces Foxp3 expression and suppressive function in induced Tregs. Eur J Immunol 2011; 41:1334-1343.

This study reports evidence that TLR7 stimulation in dendritic cells may contribute to autoimmune disease pathogenesis by causing defects in regulatory T-cell FoxP3 expression and suppressive function.

73. Bernasconi $P$, Barberis M, Baggi F, et al. Increased Toll-like receptor 4 expression in thymus of myasthenia gravis patients. Am J Pathol 2005; 167:129-139.

74. Fiola S, Gosselin D, Takada K, Gosselin J. TLR9 contributes to the recognition of EBV by primary monocytes and plasmacytoid dendritic cells. J Immunol 2010; 185:3620-3631.

75. Miyara M, Gorochov G, Ehrenstein M, et al. Human FoxP3+ regulatory T cells in systemic autoimmune diseases. Autoimmun Rev 2011; 10:744-755.

76. Kleczynska $W$, Jakiela $B$, Plutecka $H$, et al. Imbalance between Th17 and regulatory T-cells in systemic lupus erythematosus. Folia Histochem Cytobiol $2011 ; 49: 646-653$.

77. Niu Q, Cai B, Huang ZC, et al. Disturbed Th17/Treg balance in patients with rheumatoid arthritis. Rheumatol Int 2011. [Epub ahead of print]

78. Ferraro A, Socci $C$, Stabilini A, et al. Expansion of Th17 cells and functional defects in T regulatory cells are key features of the pancreatic lymph nodes in patients with type 1 diabetes. Diabetes 2011; 60:2903-2913.
79. Masuda M, Matsumoto $M$, Tanaka $S$, et al. Clinical implication of peripheral CD4+CD25+ regulatory T cells and Th 17 cells in myasthenia gravis patients. J Neuroimmunol 2010; 225:123-131.

80. Aricha R, Mizrachi K, Fuchs S, Souroujon MC. Blocking of IL-6 suppresses

I. experimental autoimmune myasthenia gravis. J Autoimmun 2011; 36:135141.

This study demonstrates that administration of anti-IL-6 antibodies to myasthenic rats downregulates several Th17-related genes without affecting the number of Treg cells. The authors propose a multifaceted mechanism of action for IL-6 blockade in EAMG, including suppression of Th1, Th17 and B-cell responses, and suggest that IL-6 and IL-6-dependent signalling are potential targets for myasthenia gravis treatment.

81. Nessi V, Nava S, Ruocco $C$, et al. Naturally occurring CD4+CD25+ regulatory $\mathrm{T}$ cells prevent but do not improve experimental myasthenia gravis. J Immunol 2010; 185:5656-5667.

82. Roche JC, Capablo JL, Larrad L. Increased serum interleukin-17 levels in patients with myasthenia gravis. Muscle Nerve $2011 ; 44: 278-280$.

83. $\mathrm{Na} \mathrm{SJ}$, So SH, Lee KO, Choi YC. Elevated serum level of interleukin-32 $\alpha$ in the patients with myasthenia gravis. J Neurol 2011; 258:1865-1870.

84. Chen $Z$, Luo $H$, Peng $Y$, et al. Comparative clinical features and immune responses after extended thymectomy for myasthenia gravis in patients with atrophic versus hyperplastic thymus. Ann Thorac Surg 2011; 91:212-218.

85. Maddison $\mathrm{P}$, McConville J, Farrugia $\mathrm{ME}$, et al. The use of rituximab in myasthenia gravis and Lambert-Eaton myasthenic syndrome. J Neurol Neurosurg Psychiatry 2011; 82:671-673.

86. Blum $\mathrm{S}$, Gillis $\mathrm{D}$, Brown $\mathrm{H}$, et al. Use and monitoring of low dose rituximab in myasthenia gravis. J Neurol Neurosurg Psychiatry 2011; 82:659-663.

87. Díaz-Manera J, Martínez-Hernández $\mathrm{E}$, Querol L, et al. Long-lasting treatment

- effect of rituximab in MuSK myasthenia. Neurology 2012; 78:189-193.

This study showed that rituximab produced long-lasting benefit in MuSK-myasthenia gravis patients. The authors recommended rituximab as an early therapeutic option for MuSK-myasthenia gravis patients if they do not respond to prednisone.

88. Gomez AM, Vrolix K, Martínez-Martínez $P$, et al. Proteasome inhibition with bortezomib depletes plasma cells and autoantibodies in experimental autoimmune myasthenia gravis. J Immunol 2011; 186:2503-2513.

89. Christadoss $P$, Tuzun E, Li J, et al. Classical complement pathway in experimental autoimmune myasthenia gravis pathogenesis. Ann N Y Acad Sci 2008; 1132:210-219.

90. Howard J, Barhon R, Freimer M, et al. Randomized, double-blind, placebocontrolled, crossover, multicenter, Phase II study of eculizumab in patients with refractory generalized myasthenia gravis [abstract]. Neurology 2012; 78:S35.0054 